\title{
Analysis of autonomic activity during head-up tilt in patients with lone paroxysmal atrial fibrillation
}

\begin{abstract}
The autonomic nervous system (ANS) has been recognized as an important modulator in the pathogenesis of paroxysmal atrial fibrillation (PAF). Changes in ANS control of heart rate (HR) and blood pressure (BP) variability occur during orthostatism to maintain cardiovascular homeostasis. The wavelet transform (WT) has been used as a tool that provides a time-frequency decomposition of the signal under investigation, allowing intermittent components to be elucidated. Aim: To study HR and BP variability with WT analysis in younger patients (P) with PAF and normal echocardiographic findings, and in healthy individuals (HI) during head-up tilt (HUT). Methods: 17P with lone PAF (age $<60$ years [33-59], 9 men) and $17 \mathrm{HI}(<60$ years [20-59], 8 men) underwent passive HUT $\left(70^{\circ}\right)$ while on sinus rhythm. Continuous monitoring of ECG and BP was obtained without antiarrhythmic drug medication. Acute changes in RR-intervals and systolic BP were evaluated with WT, and LF (low-frequency power), HF (high-frequency power) and LF/HF calculated for 1) the last 2 minutes of the supine period; 2) the 15 seconds of tilting movement (TM); 3) 1st and 2nd minutes of HUT. Results: RR intervals were identical for the two groups in baseline and during HUT. Supine basal BP was similar for both groups. During HUT, two BP response profiles were observed: a sustained increase in PAF P, and a decrease followed by an increase and further recovery in HI. WT analysis of RR intervals for PAF P, when compared to HI, showed lower LF/HF values in the supine position $(2.0 \pm 0.5$ vs. $2.5 \pm 0.3, \mathrm{p}<0.05)$, and a decreased LF power during TM $\left(574 \pm 134 \mathrm{~ms}^{2}\right.$ vs. $\left.1347 \pm 393 \mathrm{~ms}^{2}, \mathrm{p}<0.05\right)$. In the 1 st and 2 nd minutes of HUT, changes were comparable in PAF P and in HI. Analysis of systolic BP for PAF P, compared to HI, showed statistically significant differences regarding lower HF values in basal $\left(0.69 \pm 0.14 \mathrm{~ms}^{2}\right.$ vs $1.05 \pm 0.13 \mathrm{~ms}^{2}$, $\mathrm{p}<0.05)$ and lower LF and LF/HF values during TM $\left(6.3 \pm 2.5 \mathrm{~ms}^{2}\right.$ vs $8.2 \pm 1.6 \mathrm{~ms}^{2}, 8.4 \pm 2.9$ vs $12.8 \pm 4.4 ; \mathrm{p}<0.05)$. No differences were detected with respect to the 1 st and 2 nd minutes of HUT. Conclusion: P with PAF presented modified autonomic control of HR and BP in the supine position and during the initial phase of orthostatism. These findings suggest that wavelets analysis may provide a new insight into the assessment of the dynamicity of autonomic cardiovascular regulation and underscore the presence of ANS disturbances in PAF.
\end{abstract}

Keywords: atrial fibrillation, autonomic nervous system, head-up tilt, wavelet transform
Volume 13 Issue 3 - 2020

\author{
Mário Oliveira, ${ }^{1,2}$ Sérgio Laranjo, ${ }^{1,2}$ Sofia \\ Silva,' Sandra Alves,' Isabel Rocha, ${ }^{2}$ Rui Cruz \\ Ferreira' \\ 'Department of Cardiology, Santa Marta Hospital, Portugal \\ ${ }^{2}$ Institute of Physiology of Medical Faculty, University of Lisbon, \\ Portugal
}

Correspondence: Mário Oliveira, Cardiology Department, Santa Marta Hospital, Rua Santa Marta, I I69-024 Lisboa, Portugal, Tel 35I.2135943 II, Fax 351213560368, Emailm.martinsoliveira@gmail.com

Received: April 04, 2020 | Published: June 03, 2020
Abbréviations: ANS, autonomic nervous system; PAF, paroxysmal atrial fibrillation; HR, heart rate; BP, blood pressure; Wt, wavelet transform; HI, healthy individuals; HUT, head-up tilt; TM, tilting movement; AF, atrial fibrillation, RRI, R-R intervals

\section{Introduction}

Atrial fibrillation (AF) is the most common sustained cardiac arrhythmia in clinical practice. Its prevalence increases with age, having impact on both life expectancy and quality of life. ${ }^{1-4}$ The occurrence of paroxysmal AF (PAF) represents around 30\% of all $\mathrm{AF}$ cases, and is more frequent in younger patients without underlying identifiable heart disease..$^{5-7}$ Mechanisms underlying PAF pathophysiology remain a considerable challenge. The relative contribution of the multiple wavelet hypothesis, ${ }^{8}$ the focal sources of electrical activity, ${ }^{9}$ and the electrical rotors ${ }^{10}$ in explaining PAF remains unknown. Autonomic nervous system (ANS) has important modulating effects on electrophysiological properties related with atrial vulnerability for AF, with acute fluctuations of vagal and/or sympathetic activation being determinant for the occurrence of AF episodes..$^{11,12}$
During orthostatic stress, dynamic changes in autonomic control of heart rate (HR) and blood pressure (BP) variability occur to maintain cardiovascular homeostasis. These rapid short-term adjustments are mediated by the neural pathways of the ANS. ${ }^{13}$ The spectral analysis of HR and BP variability have been shown to provide indirect estimates of sympathovagal cardiovascular activity. ${ }^{14}$ Wavelet Transform has emerged as an useful tool, that provides a timefrequency decomposition of the signal under investigation, enabling transient or intermittent components to be elucidated..$^{15}$ In the present study, we used the application of wavelet analysis to HR and systolic BP signals to characterize acute ANS modulation of cardiovascular activity recorded during head-up tilting (HUT) in lone PAF patients.

\section{Subjects and methods}

The study enrolled 17 patients ( 8 females and 9 males, age $<60$ years [33-59]) affected by $\geq 1$ year of clinical history of PAF episodes, and 17 healthy volunteers (HI) ( 9 females and 8 males, $<60$ years [20-59]). PAF was documented with electrocardiogram (ECG) and/or Holter recordings. Patients with PAF were diagnosed as having lone $\mathrm{AF}$ (without clinical or echocardiographic evidence 
of cardiopulmonary disease). None of these subjects had clinical history of syncope, myocardial infarction, congestive heart failure or diabetes mellitus. Prior to the HUT, all antiarrhythmic drugs were withdrawn for at least 5 half-life times. Patients under amiodarone stopped treatment 4 weeks before the test. Alcohol and tobacco were not allowed in the day of the test. No individuals underwent tilt testing while taking medication with anticholinergic properties. Tests were performed in a quiet environment, with controlled temperature and humidity, during the morning, following four hours of fasting. ECG and BP were monitored continuously using a Task Force Monitor (CN systems, Graz, Austria). Studies were approved by the local ethics and performed after written informed consent.

\section{Head-up tilt protocol}

Subjects were placed on a tilt table with a foot plate support, secured with snug restraints (to prevent falling if syncope occurred). After a rest period of 10 minutes in the supine position, the subject was tilted to a $70^{\circ}$ head-upright position, at a constant speed within 15 seconds. Subjects were instructed to breath normally. Intravenous cannulation was not used before or during the test. Wavelet analysis was implemented on R-R intervals (RRI) and systolic blood pressure (SPB), derived from continuous monitoring of ECG and arterial $\mathrm{BP}$, respectively (see signal acquisition and processing below). The RRI and SPB data analysis was performed on four periods: 1) the last 2 minutes of the resting period (basal); 2) during the 15 seconds involved the change from supine to standing position (TM), 3) at $70^{\circ}$, during the first minute of tilt adaptation (TT1); and 4) during the second minute of head-up tilting (TT2). In each of these 1-minute periods, data analysis was performed in windows of 10 seconds. The period of 10 seconds that had the largest change from maximum to minimum was the one chosen to compare with control.

\section{Signal acquisition and processing}

Data were acquired at $1 \mathrm{Kz}$ and analysed on the time-frequency domain using the discrete wavelet transform. We selected the Daubechies 12 (Db12) wavelet because the shape of this particular wavelet resembles the type of feature present in the time series of the signal information. ${ }^{16}$ Data were then processed in an Origin environment (OriginLab, Origin Lab Scientific Graphing and Analysis Software, Origin Lab Corporation, Northampton, MA, USA). A peakto-peak routine was implemented to detect ECG and BP peaks to reconstruct the time evolution curves of RRI and SBP, which were computed in a Matlab environment (MathWorks, Natick, MA, USA). The RRI and SPB were interpolated by cubic spline and resampled (resampling period $=0.193$ ) to ensure that the centre of the frequency range of interest matched the central frequency associated to one of the scales of the DWT analysis.

Briefly, the resampled RRI and SPB time series were decomposed $(\mathrm{fRR}(\mathrm{t})$ and $\mathrm{f} \operatorname{SPB}(\mathrm{t}))$ into a sum of details and approximation at different scales of resolution. The central frequency associated with each scale ( $\mathrm{fc}$ ) was calculated by $\mathrm{fc}=\mathrm{Fc} / \mathrm{a} \Delta$, where $\mathrm{Fc}$ is the central frequency of the used wavelet (Db12), a is equal to 2-j, where the scale is $\mathrm{j}$ and $\Delta$ is the resampling period. The signal was decomposed in 12 scales. The wavelet transform of the analysed signals at scale $j$ and position $\mu$ was computed using the relation

$+\alpha W f(\mu, j)=\int f(t) 1 / \sqrt{ } 2 j \psi(t-\mu / 2 j)-\alpha$

where $\psi$ is a wavelet function, $\mathrm{Wf}(\mu, \mathrm{j})$ are the wavelet coefficients at scales and $f(t)$ either $f R R(t)$ or $f \operatorname{SPB}(t)$.
The selected wavelet coefficients for each detail related to signal frequencies between $0.038-0.15 \mathrm{~Hz}(\mathrm{LF})$ and $0.15-0.6 \mathrm{~Hz}(\mathrm{HF}) .{ }^{17}$ For the wavelets applied to the continuous SBP signal we considered only the LF values because the meaning of the HF-band in the analysis of SPB variability stills controverse and difficult to validate. The square of the details amplitude (SqA) was then calculated and, for a certain interval of time, the representative value of $\mathrm{LF}$ and $\mathrm{HF}$ was considered as the average of $\mathrm{SqA}$ across the details associated with the frequency ranges of interest.

\section{Statistical analysis}

Student's t test and repeated ANOVA were utilised for the analysis of continuous variables (overall comparison). Pair-wise comparisons of LF, HF and LF/HF between the mean of control values and the mean individual period were assessed by the Bonferroni test. The Chisquare test was used to evaluate the differences in categorical variables of two groups. $\mathrm{P}<0.05$ was considered statistically significant. Data were analyzed using GraphPAD Prism 8.0 Instruments.

\section{Results}

\section{Patient characteristics}

Seventeen patients with lone PAF were compared with a control group of HI (Table 1). There were no significant differences in age, sex ratio, arterial $\mathrm{BP}$ and $\mathrm{HR}$.

Table I Clinical characteristic of the subjects

\begin{tabular}{lll}
\hline \multirow{2}{*}{ Characteristic } & PAF group & HI group \\
\cline { 2 - 3 } & HI group & $(\mathbf{n}=17)$ \\
\hline Age & 48 & 49 \\
Years & $9 \pm 13$ & $1 \pm 7$ \\
Male gender & $53 \%$ & $47 \%$ \\
Heart rate (bpm) & $60 \pm 7$ & $62 \pm 8$ \\
$\begin{array}{l}\text { Systolic blood pressure } \\
\begin{array}{l}\text { Diastolic blood pressure } \\
(\mathrm{mmHg})\end{array}\end{array}$ & $124 \pm 18$ & $117 \pm 10$ \\
\hline
\end{tabular}

*Data with the plus/minus sign are the mean $\pm S D, p=N S$. bpm, beats per minute, PAF, paroxysmal atrial fibrillation; $\mathrm{HI}$, healthy individuals

\section{Head-up tilt}

Heart rate and blood pressure changes: All participants completed the protocol of the study without symptoms, AF initiation or fainting. During HUT, HI showed, initially, a non-significant fall in BP, followed by a return to baseline and a further increase, accompanied by an increase in HR (Table 2 and Figure 1). In the group of patients with PAF, mean arterial pressure values increased significantly during the TM, TT1 and TT2 periods ( $p<0,01$; comparing basal with the other periods), also followed by an increase in HR ( $<<0,001$; comparing basal with TT1 and TT2). Two BP response profiles (compared with the baseline) were observed: a sustained increase in PAF patients, and a decrease followed by an increase and further recovery in HI. No significant differences were observed between the two groups for HR response to the HUT during all analyzed periods (Figure 1B). Also, basal supine BP was similar for both groups (Figure 1A). However, the average systolic and diastolic BP increased immediately after the tilt in the PAF group, with mean arterial pressure values significantly higher during the HUT (Figure 1A). 
Table 2 Changes in RR intervals and blood pressure on head-up tilt (paroxysmal atrial fibrillation patients compared with the healthy volunteers group)

\begin{tabular}{|c|c|c|c|c|}
\hline & Basal & TM & TAI & TA2 \\
\hline \multicolumn{5}{|c|}{ RR intervals (ms) } \\
\hline PAF & $1014 \pm 167$ & $980 \pm 180$ & $933 \pm 180$ & $919 \pm 179 \S$ \\
\hline $\mathrm{HI}$ & $963 \pm 117$ & $974 \pm 125$ & $923 \pm 120 *$ & $885 \pm 125^{*}$ \\
\hline \multicolumn{5}{|c|}{ Systolic blood pressure $(\mathrm{mmHg})$} \\
\hline PAF & $124 \pm 18$ & $129 \pm 19$ & $135 \pm 17^{*}$ & $135 \pm 12^{*}$ \\
\hline $\mathrm{HI}$ & $117 \pm 10$ & $115 \pm 11$ & $115 \pm 10$ & $120 \pm 8$ \\
\hline \multicolumn{5}{|c|}{ Diastolic blood pressure $(\mathrm{mmHg})$} \\
\hline PAF & $81 \pm 12$ & $86 \pm 12^{*}$ & $95 \pm 13^{*}$ & $96 \pm 10 *$ \\
\hline $\mathrm{HI}$ & $77 \pm 6$ & $74 \pm 9$ & $78 \pm 10$ & $8 I \pm 9 * *$ \\
\hline \multicolumn{5}{|c|}{ Mean blood pressure $(\mathrm{mmHg})$} \\
\hline PAF & $96 \pm 13$ & $101 \pm 13^{*}$ & $109 \pm 14^{*}$ & $109 \pm 10 *$ \\
\hline $\mathrm{HI}$ & $90 \pm 10$ & $88 \pm 9$ & $90 \pm 10$ & $94 \pm 8 * *$ \\
\hline
\end{tabular}

PAF, paroxysmal atrial fibrillation group; $\mathrm{HI}$, healthy individuals group

${ }^{*} \mathrm{p} \leq 0,0 \mathrm{I}$; **p $=0,02 ; \S=\mathrm{p}<0,00$ I, when compared to basal. Data are expressed as mean $\pm S D$

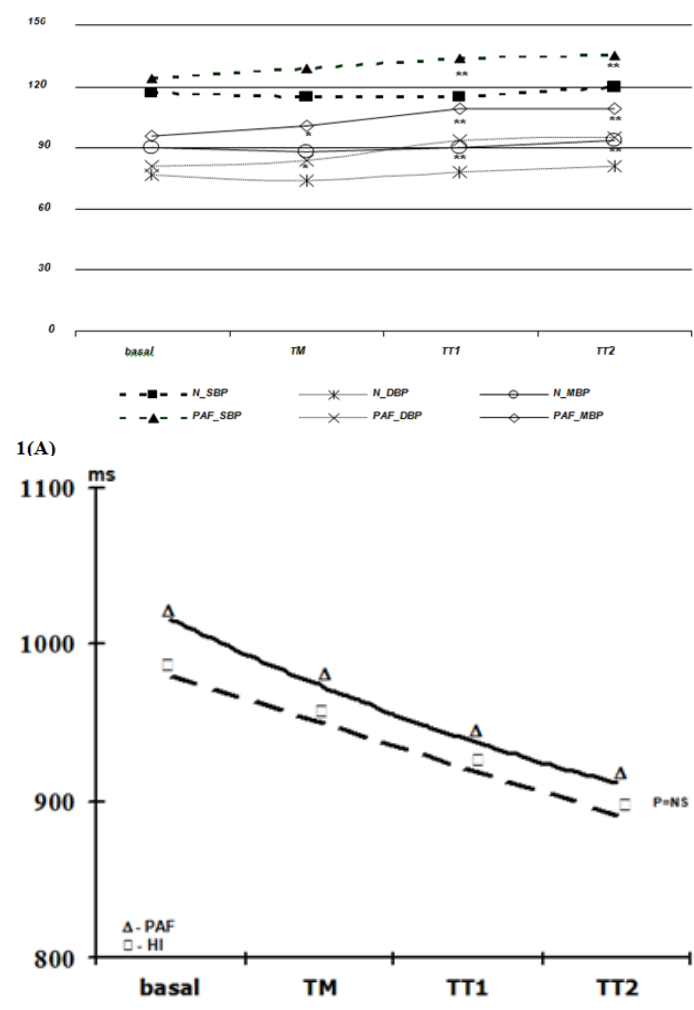

1(B)

Figure I Blood pressure (IA) and heart rate (IB) responses during head-up tilting in patients with paroxysmal atrial fibrillation and in healthy individuals. basal=supine position; TM=during the 15 seconds of tilting movement; TTI=during the first minute of tilting $\left(70^{\circ}\right)$; TT2=during the second minute of tilting $\left(70^{\circ}\right)$; PAF_SBP, PAF_MBP and PAF_DBP=systolic, mean and diastolic blood pressure in paroxysmal atrial fibrillation patients, respectively; N SPB, N_MBP and N_DBP=systolic, mean and diastolic blood pressure in healthy volunteers, respectively. Data expressed as mean values. ( $p=N S$ between $\mathrm{HI}$ and PAF patients for basal blood pressure and for RR intervals in each period; $*=\mathrm{p} \leq 0,01 ; * *=\mathrm{p} \leq 0,001)$.

\section{Heart rate and blood pressure variability}

HR and SBP variability were significantly different in patients with PAF compared to HI, during supine rest and in HUT.

Wavelet analysis of RRI showed a significant increase of the LF band during the TM and TT1 periods (compared to basal), and a significant decrease of the HF band in the TT2 period for the HI group, while, in the PAF group, an increase of the LF band was observed only in the TT1 period without no significant changes in the $\mathrm{HF}$ band (figure 2). At TM, the LF values were significantly lower in the PAF group than in HI (574 134 ms2 vs. $1347 \pm 393 \mathrm{~ms} 2 ; \mathrm{p}<0,05)$. In basal, the LF/HF ratio was lower in the PAF group $(2,01 \pm 0,52$ vs. $2,55 \pm 0,34, \mathrm{p}<0,05)$, followed by a significant sustained increased in both groups (from $4,5 \pm 1,5$ after orthostasis to $12,2 \pm 2,9$ during TT2 in PAF, and from 3,29 $\pm 0,67$ after orthostasis to $9,74 \pm 3,68$ during TT2 in HI, p=NS) (Figure 2). Analysis of SBP for PAF patients showed statistically significant differences regarding lower HF values in basal compared to $\mathrm{HI}\left(0.69 \pm 0.14 \mathrm{~ms}^{2}\right.$ vs. $\left.1.05 \pm 0.13 \mathrm{~ms}^{2}, \mathrm{p}<0.05\right)$ (figure 3). During TM, LF and LF/HF values were also lower in the PAF group $\left(6.3 \pm 2.5 \mathrm{~ms}^{2}\right.$ vs. $8.2 \pm 1.6 \mathrm{~ms}^{2}, 8.4 \pm 2.9$ vs $12.8 \pm 4.4$; $\left.\mathrm{p}<0.05\right)$. No differences were detected with respect to the TA1 and TA2 periods.
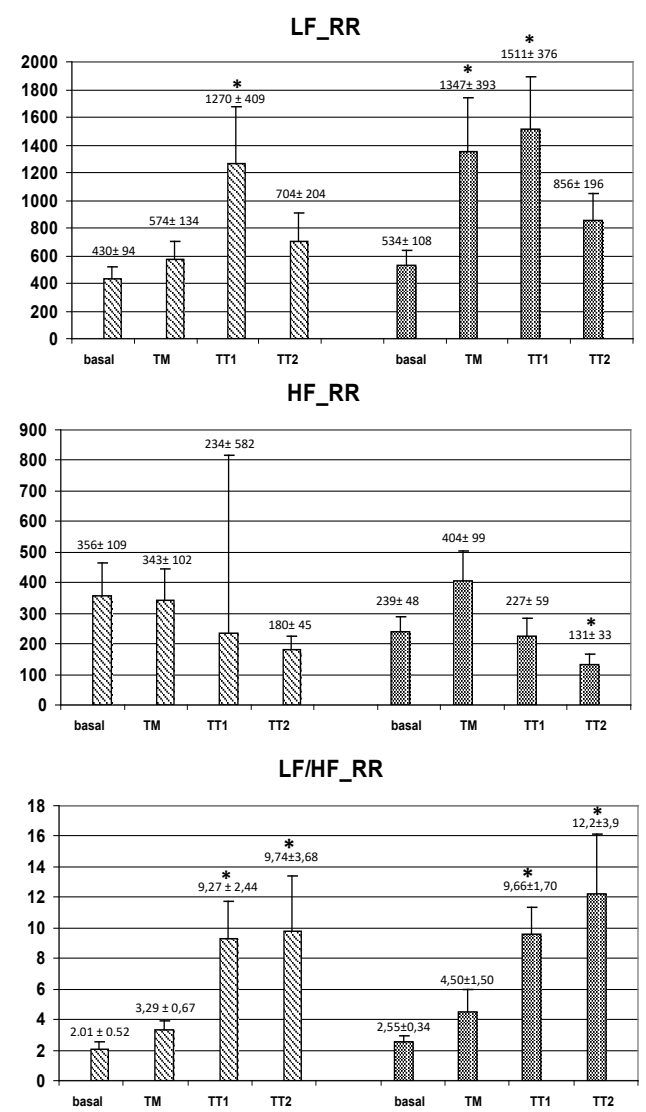

Figure 2 Changes in autonomic outflow parameters obtained from the RR intervals during head-up tilt. basal=supine position; $\mathrm{TM}=$ during the 15 seconds of tilting movement; $\mathrm{TTI}=$ during the first minute of tilting $\left(70^{\circ}\right) ; \mathrm{TT} 2=$ during the second minute of tilting $\left(70^{\circ}\right)$; $\mathrm{PAF}=$ paroxysmal atrial fibrillation; $\mathrm{HI}=$ healthy individuals; $L F \_R R=L F$ band from RR intervals; HF_RR=HF band from $R R$ intervals; $L F / H F-R R=L F / H F$ ratio from $R R$ intervals. Data expressed as mean \pm SEM. ( $p<0,05$ between $\mathrm{HI}$ and PAF patients for basal LF/HF and for LF during TM; $*=p<0,05$ when compared to basal).

\section{Discussion}

This study compared, by using wavelet transforms analysis, the ANS activity involved in the acute adaptation of HR and BP during 
HUT, in HI and PAF patients. The results have demonstrated that, despite similar clinical characteristics and no differences of mean HR and $\mathrm{BP}$ in basal conditions, patients with PAF have different values for autonomic outflow in the supine condition, with occurrence of significant differences in the analysis of the ANS responses during the initial period of adaptation after orthostasis.

The wavelet transform has emerged as a useful tool that provides a time-frequency decomposition of nonstationary signals under investigation, which allows intermittent components to be elucidated. Wavelet analysis is able to reflect a precise, temporally localized, shift in ANS equilibrium. ${ }^{17}$ This method has been shown to be applicable in short duration analysis, allowing the visualization in time of the contribution of LF and HF to the observed dynamic changes of a particular signal. ${ }^{15,16}$ It is especially valuable because of its ability to elucidate simultaneously spectral and temporal information. Wavelet analysis has been widely accepted for performing time-frequency decomposition of cardiovascular signals. ${ }^{17-19}$ There are several types of wavelet functions to be used for the analysis of physiological signals. In this study, the Daubechie type was selected because it has been proposed as the most suitable for BP and HR signal profiles. ${ }^{15,16}$ We studied the contribution of two sets of frequencies, LF-band $(0,038-0,15 \mathrm{~Hz})$ and HF-band $(0,15-0,60 \mathrm{~Hz})$, to acute changes in RRI and SBP evoked by HUT.

\section{Physiological effects of the head-up tilt}

Changes in autonomic control of HR and BP variability occur during orthostatic stress to maintain cardiovascular homeostasis. The use of non-invasive methodologies to evaluate ANS function, based in frequency domain methods, have demonstrated that analysis of RRI and SBP variability constitutes one of the most widespread tools used to investigate on the coupling mechanisms underlying shortterm cardiovascular regulation. ${ }^{20}$ HUT is a physiological stimulus that is associated with increased sympathetic tone and withdrawal of vagal activity. ${ }^{21}$ Classically, HUT causes two phases of adaptation: an earlier cardiovascular response, that can be observed during the first 30 seconds after orthostasis, followed by a stabilization period, composed by an early adaptation occurring in the first 1-2 minutes of HUT and a second period related to prolonged orthostasis, that lasts more than 5minutes. ${ }^{15,16,22}$ The rapid short-term adjustments to orthostatic stress are mediated by the neural pathways of the ANS. Therefore, our results show, by using the wavelet transform analysis applied to the four periods under observation, the modifications of ANS activity underlying HR and BP changes.

The PAF group had lower basal LF/HF for the HRV analysis, and lower HF for the SBP variability. These observations may represent an exaggerated feedback interaction occurring in the direction from SBP to RR interval. It is widely accepted that the sympathetic influences are better seen on the LF band of BP signal whereas the parasympathetic activity is better expressed on HF band of HR recording. ${ }^{15,16}$ Moreover, an ANS balance in favour of the cardiac influence of vagal activity, with a lower influence of the parasympathetic outflow in the arterial system in the supine position, might be interpreted as a tendency to an enhanced cardiac vagal activity, and a more direct arterial effect of sympathetic nervous system in PAF patients. In fact, it is well known that vagal nerve stimulation can result in significant changes in cardiac electrophysiology that predispose patients to develop paroxysmal atrial arrhythmias..$^{12,23,24}$ Nevertheless, the observation of a lower basal HF in the analysis obtained from the SBP variability is difficult to explain, because the meaning of the HF-band in the analysis of
SPB variability stills controversial and difficult to validate. In fact, HF power of BP variability does not have an autonomic correlate, but rather, it corresponds to the mechanical effects of respiration that may act directly on the pressure gradients of intrathoracic vessels. ${ }^{14}$ Furthermore, the focus of this study was the analysis of the ANS activity during the adaptation phases to HUT.

In HUT, where an orthostatic challenge is performed, HI showed a significant sustained increase of sympathetic flow derived from RRI and SPB starting during TM, while in PAF patients, this increment occurred later, during the TT1 period. Also, HI had a higher value for $\mathrm{LF} / \mathrm{HF}$ ratio obtained from the SBP, since the beginning of the orthostatism (Figure 3), indicating a different balance between sympathetic and parasympathetic outflows. Strictly speaking, HRV and SBP variability represent measures of the heart's response, and the blood vessels' response, to the autonomic outflow. Therefore, the present results support the notion that patients with lone PAF may have abnormalities in the ANS function, has proposed by other authors. ${ }^{25,26}$

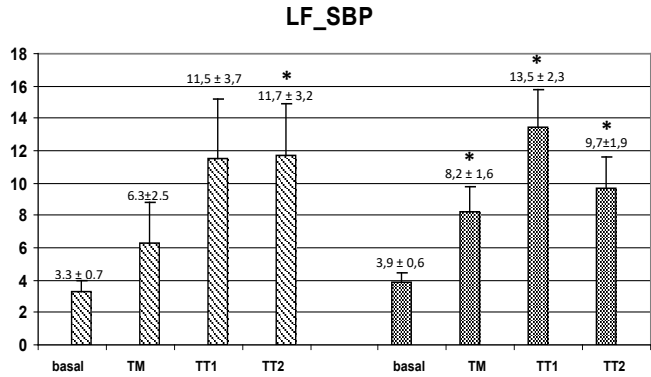

HF_SBP

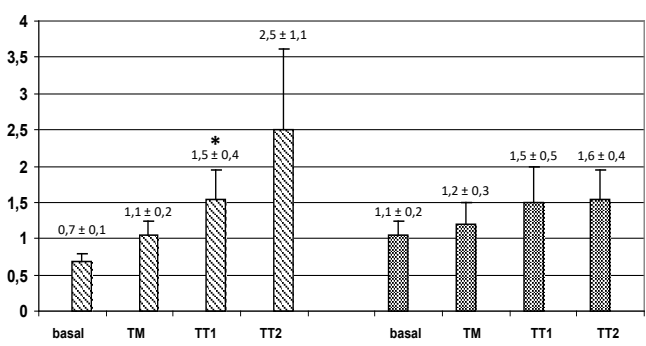

LF/HF_SBP

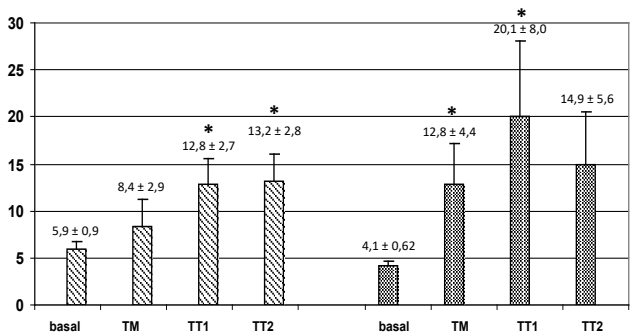

Figure 3 Changes in autonomic outflow parameters obtained from the systolic blood pressure during head-up tilt. basal=supine position; TM=during the 15 seconds of tilting movement; TTI=during the first minute of tilting $\left(70^{\circ}\right)$; $\mathrm{TT} 2=$ during the second minute of tilting $\left(60^{\circ}\right)$; PAF=paroxysmal atrial fibrillation; $\mathrm{HI}=$ healthy individuals; $L F \_S B P=L F$ band from systolic blood pressure; HF_SBP=HF band from systolic blood pressure; LF/HF_RR=LF/HF ratio from systolic blood pressure. Data expressed as mean $\pm S \overline{E M} .(p<0,05$ between $\mathrm{HI}$ and PAF patients for basal HF and for LF and LF/HF during TM; $*=p<0,05$ when compared to basal).

The differences detected in the temporal changes of ANS activity in PAF patients occurred, however, with significant higher values for the diastolic and mean BP during the TM period, associated with 
higher SBP during TT1 and TT2, despite similar heart rates. These observations could be related with a progressive adaptation of the autonomic cardiovascular receptors due to a certain degree of ANS dysfunction. In fact, abnormalities of the autonomic tone can lead to an up-regulation of the cardiovascular receptors, which may be related with a heightened susceptibility to autonomic cardiovascular responses. ${ }^{27,28}$ Thus, it could be that the delay observed in the increase of the sympathetic activity for RRI and SBP variability reflects an abnormality of the ANS outflow, whereas the higher BP values with similar RRI during orthostasis analysis may be a result of an increased cardiovascular sensitivity to ANS influences. Also, a complementary view in the interpretation of these data may be in concordance with previous studies showing that essential hypertension is linked to basic alterations in cardiovascular autonomic control, with specific abnormalities being already present at a very early stage of the disease, perhaps even before any sign of increased BP can be observed. ${ }^{29}$ Akselrod and coworkers showed that mild-hypertensive subjects displayed a marked reduction in the rise of LF-band fluctuations in BP variability during postural changes. ${ }^{30}$ It is well known, from prospective surveys, that systemic hypertension is the most common disease associated with all types of AF. ${ }^{31-32}$ Therefore, changes of ANS responses detected during HUT, might also be looked as a potential indicator for the development of hypertension in the future. The physiological meaning of these results, reflecting a complex interaction between adrenergic and vagal tone in PAF patients, represents a challenge that needs to be further explored, both as a contribution to the understanding of the mechanisms underlying PAF and as a marker of clinical outcome.

\section{Study limitations}

First, the protocol did not include HR and SBP variability measurements with wavelet analysis beyond the 2 first minutes of orthostatic stress. Therefore, comparison of the autonomic activity occurring during prolonged standing cannot be discussed in this study. Nevertheless, it is accepted that the rapid short-term adjustments in the first two minutes of HUT are mediated by the neural pathways of the ANS. Another limitation lies on the fact that, although the number of subjects included in the study allowed for the identification of significant differences in the parameters considered for the evaluation of ANS function and BP responses during HUT in the group with PAF patients, the resulting sample was relatively small. Thus, further studies in a larger group may be needed to confirm these findings.

\section{Conclusion}

Patients with lone PAF present modified autonomic control of HR and SBP in the supine position and during the initial phase of HUT. These findings underscore the presence of ANS disturbances in PAF, and indicate that wavelet analysis allows the evaluation of acute and transient changes of HR and SBP variability, providing a new insight into the assessment of autonomic cardiovascular function in clinical practice.

\section{Acknowledgments}

None.

\section{Conflicts of interest}

The authors declare there are no conflicts of interest related to the article.

\section{Funding}

None.

\section{References}

1. Chugh SS, Havmoeller R, Narayana K, et al. Worldwide epidemiology of atrial fibrillation: a Global Burden of Disease 2010 Study. Circulation. 2014;129(8):837-847.

2. Feinberg WM, Blackshear JL, Laupacis A, et al. Prevalence, age distributions, and gender of patients with atrial fibrillation: Analysis and implications. Arch Intern Med. 1995;155:469-473.

3. Benjamin EJ, Wolf PA, D'Agostino RB, et al. Impact of atrial fibrillation on the risk of death. The Framinghan Heart Study. Circulation. 1998;98(10):946-952.

4. Van Den Berg MP, Hassink RJ, Tuinenburg AE, et al. Quality of life in patients with paroxysmal atrial fibrillation and its predictors: importance of the autonomic nervous system. Eur Heart J. 2001;22(3):247-253.

5. Seet RA, Friedman PA, Rabinstein AA. Prolonged rhythm monitoring for the detection of occult paroxysmal atrial fibrillation in ischemic stroke of unknown cause. Circulation. 2011;124(4):477-486.

6. Nieuwlaat R, Capucci A, Camm AJ, et al. European Heart Survey Investigators. Atrial fibrillation management: a prospective survey in ESC member countries: the Euro Heart Survey on Atrial Fibrillation. Eur Heart J. 2005;26(22):2422-2434.

7. Filippi A, Bettoncelli, Zaninelli A. Detected atrial fibrillation in North Italy: rates, calculated stroke risk and proportion of patients receiving thrombo-prophylaxis. Family Practice. 2000;17(4):337-339.

8. Moe GK, Rheinboldt WC, Abilddskov JA. "A computer model of atrial fibrillation”. Am Heart J. 1964;67(2):200-220.

9. Haissaguerre M, Jais P, Shah DC. Spontaneous initiation of atrial fibrillation by ectopic beats originating in the pulmonary veins. $N$ Engl $J$ Med. 1998;339:659-666.

10. Narayan SM, Krummen DE, Rappel WJ. Clinical mapping approach to diagnose electrical rotors and focal impulse sources for human atrial fibrillation. J Cardiovasc Electrophysiol. 2012;23(5):447-454.

11. Chen P, Tan AY. Autonomic nerve activity and atrial fibrillation. Heart Rhythm. 2007;4(3 Suppl):S61-S64.

12. Oliveira M, Postolache G, Geraldes V, et al. Acute electrophysiological modulation of the atria and pulmonary veins: Effects of sympathetic and parasympathetic interaction on atrial fibrillation inducibility. Revista Portuguesa de Cardiologia. 2012;31(3):215-223.

13. Wieling W, van Lieshout JJ. Maintenance of postural normotension in humans. In: Low PA. Clinical Autonomic Disorders. Evaluation and Management. Boston: Little Brown; 1993: p. 69-77.

14. Pagani M, Lombardi F, Guzzetti S, et al. Power spectral analysis of heart rate and arterial pressure variabilities as a marker of sympathovagal interaction in man and conscious dog. Circ Res. 1986;59:178-193.

15. Ducla-Soares J, Santos-Bento M, Laranjo S, et al. Wavelet analysis of autonomic outflow of normal subjects on head-up tilt, cold pressor test, Valsalva Manouevre and deep breathing. Experimental Physiology. 2007;92(4):677-686.

16. Postolache G, Rocha I, Silva-Carvalho L, et al. A practical approache of wavelets analysis to follow transitory modulation of the cardiac autonomic system after ethanol administration. IEEE IMT. 2003;218-222.

17. Busso JC, Roche F, Costes F, et al. Wavelet transform to quantify heart rate variability and to assess its instantaneous changes. $J$ Appl Physiol. 1999;86:1081-1091. 
18. Toledo E, Gurevitz O, Hod H, et al. Wavelet analaysis of instantaneous heart rate: a study of autonomic control during thrombolysis. $A M J$ Physiol Regul Integr Com Physiol. 2003;284:R1079-R1091.

19. Verlinde D, Beckers F, Ramaekers D, et al. Wavelet decomposition analysis of heart rate variability in aerobic athletes. Auton Neurosci. 2001;90(1-2):138-141.

20. Wichterle D, Melenovsky V, Simek J, et al. Cross-spectral analysis of heart rate and blood pressure modulations. Pacing Clin Electrophysiol. 2000;23:1425-1430.

21. Ingemansson MP, Holm M, Olsson SB. Autonomic modulation of the atrial cycle length by the head up tilt test: non-invasive evaluation in patients with chronic atrial fibrillation. Heart. 1998;80:71-76.

22. Hilz MJ, Dutsch M. Quantitative studies of autonomic function. Muscle Nerve. 2006;33(1):6-20.

23. Peng-Sheng C, Tan AY. Autonomic nerve activity and atrial fibrillation. Heart Rhythm. 2007;4(3 Suppl):S61-S64.

24. Oliveira M, da Silva N, Geraldes V. et al. Acute vagal modulation of electrophysiology of the atrial and pulmonary veins increases vulnerability to atrial fibrillation. Exp Physiol. 2011;96(2):125-133.

25. Xi Y, Cheng J. Dysfunction of the autonomic nervous system in atrial fibrillation. J Thorac Dis. 2015;7(2):193-198.

26. Olshansky B. Interrelationships Between the Autonomic Nervous System and Atrial Fibrillation. Prog in Cardiov Diseases. 48(1):2005:57-78.
27. Delahaye N, Le Guludec D, Dinanian S, et al. Myocardial Muscarinic Receptor Upregulation and Normal Response to Isoproterenol in Denervated Hearts by Familial Amyloid Polyneuropathy. Circulation. 2001;104(24):2911-2916.

28. Akselrod S. Time-frequency analysis of heart rate variability under autonomic provocations: Dynamic electrocardiography. In: Marek Malic A, John Camm, editors. Armonk, NY, USA: Futura Publishing; 2004.

29. Akselrod S, Oz O, Grmberg M, et al. Autonomic response to change of posture among normal and mild-hypertensive adults: investigated by time-dependent spectral analysis. Journal of the Autonomic Nervous System. 1997;64:33-43.

30. Nieuwlaat R, Capucci A, Camm AJ, et al. Atrial fibrillation management: a prospective survey in ESC Member Countries. The Euro Heart Survey on Atrial Fibrillation. Euro Heart J. 2005;26(22):2422-2434.

31. Alan S, Elaine M, Kathleen A, et al. Prevalence of Diagnosed Atrial Fibrillation in Adults. National Implications for Rhythm Management and Stroke Prevention: the An Ticoagulation and Risk Factors in Atrial Fibrillation (ATRIA) Study. JAMA. 2001;285:2370-2375.

32. De Vos, Breithardt CB, Camm G, et al, Progression of atrial fibrillation in the REgistry on Cardiac rhythm disORDers assessing the control of Atrial Fibrillation cohort: clinical correlates and the effect of rhythm-control therapy. Am Heart J. 2012;163:887-893. 